\title{
FUSE Observations of the Dwarf Novae UU Aql, BV Cen, and CH UMa in Quiescence ${ }^{1}$
}

\author{
Edward M. Sion, Patrick Godon², Fuhua Cheng \\ Astronomy and Astrophysics, Villanova University, \\ 800 Lancaster Avenue, Villanova, PA 19085, USA \\ edward.sion@villanova.edu, patrick.godon@villanova.edu, fcheng@ast.vill.edu \\ Paula Szkody \\ Department of Astronomy, University of Washington, Seattle, WA 98195, USA \\ szkody@astro.washington.edu
}

\begin{abstract}
We report on FUSE spectra of three U Gem-type, long period, dwarf novae, UU Aql, BV Cen and CH UMa taken during their quiescence intervals. We discuss the line identifications in their spectra and attempt to characterize the source(s) of their FUV flux distribution. Archival IUE spectrum of CH UMa and BV Cen in quiescence were identified as having a matching flux level with the FUSE spectra and these were combined with each FUSE spectrum to broaden the wavelength coverage and further constrain model fits. Multi-component synthetic spectral fits from our model grids, consisting of single temperature white dwarfs, two-temperature white dwarfs, accretion disks and white dwarfs plus accretion disks, were applied to the FUSE spectra alone and to the combined FUSE + IUE spectra. We present the results of our model analyses and their implications.
\end{abstract}

Subject headings: accretion, accretion disks - novae, cataclysmic variables - stars: dwarf novae - stars: individual (UU Aql, BV Cen, CH UMa) - white dwarfs

\footnotetext{
${ }^{1}$ Based on observations made with the NASA-CNES-CSA Far Ultraviolet Spectroscopic Explorer. FUSE is operated for NASA by the Johns Hopkins University under NASA contract NAS5-32985

${ }^{2}$ Visiting at the Space Telescope Science Institute, Baltimore, MD 21218, USA; godon@stsci.edu
} 


\section{Introduction}

Dwarf novae (DNe) are close interacting binaries in which a Roche-lobe filling main sequence-like dwarf transfers matter with angular momentum through a disk onto a white dwarf (WD). The rapid disk accretion during outburst, due to a thermal instability that causes cyclic changes of the accretion rate, releases gravitational potential energy identified as the DN outburst. The high accretion rate $\left(\sim 10^{-8}\right.$ to $10^{-9} M_{\odot} /$ yr $)$ outburst phase (which lasts a few days to weeks $)$ is preceded and followed by a low accretion rate $\left(\sim 10^{-11} M_{\odot} /\right.$ yr $)$ quiescence stage. This DN behavior is believed to be punctuated every few thousand years or more by episodes of catastrophically unstable thermonuclear burning, the classical nova explosion. Perhaps the least understood topic in $\mathrm{CV} / \mathrm{DN}$ research (along with what drives the wind outflow in outburst) is the state and structure of the boundary layer and accretion disk during quiescence and the physics of how long term accretion of mass, angular momentum and energy affects the WD. Our studies with archival IUE, and HST/STIS have found that $\sim 50 \%$ of the DNe in quiescence are dominated (i.e., $>60 \%$ of UV flux) by a component of FUV flux other than the WD called the "accretion disk"; $\sim 25 \%$ are dominated by the WD and $\sim 25 \%$ have nearly equal contribution of WD and accretion disk (40-60\% each) (Urban \& Sion 2006).

A number of studies (Araujo-Betancor et al. 2003; Sion 1991, 1999; Urban \& Sion 2006) have shown that CV WDs above the gap are typically on-average 10,000K hotter than CV WDs below the period gap (almost certainly a consequence of higher timeaveraged accretion rates of systems above the gap but possibly with system total age also being a factor). Since the white dwarf surface temperature is crucial for understanding CV evolution and whether CVs evolve across the period gap, the use of cooling ages and long term evolutionary model sequences with accretion (including the effects of nova explosions, Townsley \& Bildsten (2003)) must rely on the empirical WD temperature of the photosphere in equilibrium with long term compressional heating from accretion. The work of Townsley \& Bildsten (2002, 2003) allows measured T $\mathrm{T}_{\text {eff }}$ 's of CV WDs to be converted to the accretion rate per unit WD surface area averaged over the thermal time of the WD envelope.

Unfortunately, there are far fewer systems with reliably known WD properties above the period gap compared with below the gap, thus impeding detailed comparisons between the two groups. For example, among CVs below the gap, there are now roughly 20 systems

with reliable WD temperatures but only 5 systems above the gap with reliable WD temperatures. The primary reason for this disparity is that in long period CVs with higher mass transfer rates, the disks may remain optically thick even during quiescence, making the disk contribution to the total flux typically larger in systems above the gap. Hence, it is more 
difficult to disentangle the white dwarf flux contribution from that of the accretion disk.

As part of our effort to increase the sample of CV degenerates with known properties above the gap, we have used FUSE and IUE archival spectra to analyze three long period dwarf novae, UU Aql, BV Cen, and CH UMa. For UU Aql, system properties were adopted from Ritter \& Kolb (2003) and from Szkody (1987). For BV Cen parameters were adopted from Ritter \& Kolb (2003). For CH UMa, we adopted values from Friend et al. (1990). For all three systems, the distances were the same as those in Urban \& Sion (2006) where the Warner (1995) and Harrison et al. (2004) $\mathrm{M}_{v(\max )}$ versus $\mathrm{P}_{\text {orb }}$ relations, calibrated with trigonometric parallaxes, were used. The reddening values were the same as those quoted in Urban \& Sion (2006) which were from Verbunt (1987), laDous (1991) and Bruch \& Engel (1994).

The dwarf nova systems analyzed in this work are UU Aql, BV Cen, and CH UMa. In Table 1, the observed properties of these dwarf novae are summarized by column as follows: (1) system name; (2) dwarf nova subclass with UG denoting a U Gem-type system; (3) orbital period in days; (4) the recurrence time of dwarf nova outbursts in days; (6) the apparent magnitude at minimum (quiescence); (7) the apparent magnitude in outburst; (8) secondary spectral type; (9) orbital inclination in degrees; (10) white dwarf mass in solar masses; (11) secondary star mass in solar masses; (12) adopted reddening value and ; (13) distance in parsecs.

\section{Observations and Data Reduction}

The instrumental setup and exposure details of the FUSE spectra of BV Cen, CH UMa and UU Aql in quiescence are provided in Table 2. The LWRS was used in all cases since it is least prone to slit losses due to the misalignment of the four FUSE telescopes. All the spectra were obtained in time tag (TTAG) mode, and each one of them consists of 7 individual exposures (corresponding to 7 FUSE orbits). It is clear that the relatively poor FUSE spectral quality of the spectra speaks to the requirement for more observing time. Nevertheless, we deemed that there was sufficient $\mathrm{S} / \mathrm{N}$ to warrant a first attempt multicomponent FUV analysis of each system

All the data were reduced using CalFUSE version 3.0.7. In this version of CalFUSE the data are maintained as a photon list: the intermediate data file - IDF. Bad photons are flagged but not discarded, so the user can examine and combined data without re-running CalFUSE. For each target, we combined the individual exposures (using the IDF files) and channels to create a time-averaged spectrum weighted in the flux in each output datum by 
the exposure time and sensitivity of the input exposure and channel of origin. The details are given here. The spectral regions covered by the spectral channels overlap, and these overlap regions are then used to renormalize the spectra in the $\mathrm{SiC} 1, \mathrm{LiF} 2$, and $\mathrm{SiC} 2$ channels to the flux in the LiF1 channel. We then produce a final spectrum that covers almost the full FUSE wavelength range $905-1182 \AA$. The low sensitivity portions of each channel are discarded. In most channels there exists a narrow dark stripe of decreased flux in the spectra running in the dispersion direction. This stripe has been affectionately known as the "worm" and it can attenuate as much as $50 \%$ of the incident light in the affected portions of the spectrum. The worm has been observed to move as much as 2000 pixels during a single orbit in which the target was stationary. The "worm" appears to be present in every exposure and, at this time, there is no explanation for it. Because of the temporal changes in the strength and position of the worm, CalFUSE cannot correct target fluxes for its presence. Here we take particular care to discard the portion of the spectrum where the so-called worm 'crawls', which deteriorates LiF1 longward of $1125 \AA$. Because of this the $1182-1187 \AA$ region is lost. We then rescale and combine the spectra. When we combine, we weight according to the area and exposure time for that channel and then rebin onto a common wavelength scale with a $0.1 \AA, 0.2 \AA$, and $0.5 \AA$ resolution.

In the observing log given in Table 2, the entries are by column: (1) gives the target, (2) FUSE spectral data ID, (3) the aperture used, (4) the date and time of observation, (5) the (good) exposure time in seconds, (6) central wavelength, and (7) S/N.

The FUSE spectra for the three systems, UU Aql, BV Cen and CH Uma are displayed in Figures 1, 2, and 3 respectively. A quantitative sense of the relative data quality is provided by the signal to noise for the three FUSE spectra. We binned the data by $0.1 \AA$ for which the $\mathrm{S} / \mathrm{N}$ of UU Aql, BV Cen, and CH UMa is 5.15, 5.9, and 3.6, respectively.

For UU Aql, Fig. 1 reveals a rich line spectrum with numerous lines of molecular hydrogen, interstellar species and possible accretion disk or photospheric absorption features. The spectrum reveals a downturn in the continuum shortward of $1000 \AA$. The spectrum does not exhibit any evidence of emission lines from the source, the only emission lines are from

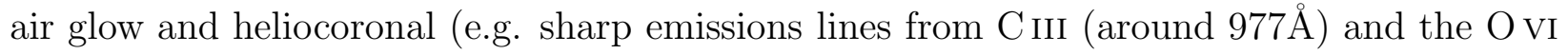
doublet).

In figure 2, the FUSE spectrum of BV Cen has a variety of interstellar and stellar features but has a continuum shape distinctly different from UU Aql. The broad CIII absorption feature around $1175 \AA$ is definitely from the source, and the CIII (around $977 \AA$ ) and the O VI doublet broad emission features could also possibly be associated with one of the FUV components in BV Cen. All the other sharp emission features are either heliocoronal or geocoronal in origin. 
In figure 3, CH UMa reveals numerous absorption lines due to highly ionized and singly ionized metals. It has a continuum energy distribution similar to UU Aql. There are definitely some broad emission lines from the source itself. The most prominent one is the $\mathrm{O}$ vi doublet (the right component being strongly attenuated by molecular hydrogen sharp absorption lines), C III (both around $977 \AA$ and $1175 \AA$ ) which also seems to be in emission and a tentative identification of N IV emission in the short wavelengths. The source is also contaminated with sharp emission lines due to air glow. Here too the N I \& N II are geocoronal in origin, and the sharp peaks on top of the broad C III (977 $)$ ) and O vi emissions are heliocoronal in origin. We note that ISM molecular hydrogen absorption is affecting the continuum.

Since the wavelength range covered by FUSE overlaps with HST/STIS or IUE in the region of $\mathrm{C}$ III between $1170 \AA$ and $1180 \AA$, a much broader FUV wavelength coverage is afforded by combining the spectra when the flux levels of the two spectra in the wavelength overlap region match closely enough. We found archival IUE spectra matching the FUSE spectra of two of the three systems (CH UMa and BV Cen) but unfortunately no HST spectra exist for the three systems.

The FUSE + IUE combination of spectra rests on the assumptions that (1) differences between the two spectra in orbital phase and (2) in time after the last outburst can be ignored. Given the long exposure times of the FUSE and IUE spectra, the questionable reliability of the orbital ephemerides, including UU Aql's and (3) the limited S/N of the quiescent spectra, the influence of phase-dependent variations is not considered. However, the time since the last outburst as well as the brightness state of the system at the times of the FUSE and IUE observations for the three systems is considered in detail using AAVSO archival light curve data. For UU Aql, the FUSE spectrum was obtained approximately 50 days after its last major dwarf nova outburst, however, the $I U E$ spectrum was obtained during the transition to a brightening that appeared not to be a major outburst. Therefore, we have excluded the IUE spectrum from use in combination with the FUSE data for UU Aql. For BV Cen both the FUSE and IUE spectra were obtained during quiescence but the FUSE spectrum was acquired approximately 159 days after the last outburst while the IUE spectrum was taken roughly 50 days after the last outburst. Thus, the FUSE spectrum probably recorded a greater degree of white dwarf cooling than the IUE spectrum obtained closer to the last outburst. Likewise for $\mathrm{CH}$ UMa, both the FUSE and IUE spectra were obtained during quiescence but the FUSE spectrum appears to have been obtained roughly 125 days after the last outburst while the IUE spectra were acquired about 83 days after the last outburst. Since the e-folding times for white dwarf cooling in both of these systems following the outburst heating episode is typically shorter than the above two post-outburst intervals, it is probably acceptable to combine the FUSE and IUE spectra for BV Cen and 
CH UMa.

In Table 3 we present the observing log of IUE observations which matched the FUSE flux level in the wavelength overlap region of these two systems. The entries by column are (1) the target name, (2) the observation ID, (3) aperture, (4) dispersion mode, (5) date of the observation, (6) time of mid-exposure, and (6) the exposure time in seconds.

Thus, our analysis was carried out first for the FUSE spectra of the three systems and then separately for the combined FUSE plus IUE data of BV Cen and CH UMa.

\section{Multi-Component Synthetic Spectral Fitting}

Our data analysis and modeling involves the full suite of multi-component (accretion disk, white dwarf photosphere, accretion belt) synthetic spectral codes, which we have utilized in our spectral fitting of FUSE and IUE data. Based upon our expectation that the accreting white dwarf is an important source of FUV flux in these systems during quiescence, we carried out a high gravity photosphere synthetic spectral analysis first. The model atmosphere (TLUSTY200; Hubeny (1988)), and spectrum synthesis codes (SYNSPEC48 and ROTIN4 Hubeny \& Lanz (1995)) and details of our $\chi_{\nu}^{2}\left(\chi^{2}\right.$ per degree of freedom) minimization fitting procedures are discussed in detail in Sion et al. (1995) and will not be repeated here. To estimate physical parameters, we generally took the white dwarf photospheric temperature $\mathrm{T}_{e f f}, \log g$, and rotational velocity $v_{r o t}$ and chemical abundances as free parameters.

We normalize our fits to 1 solar radius and 1 kiloparsec such that the distance of a source is computed from $d=1000(p c) *\left(R_{w d} / R_{\odot}\right) / \sqrt{S}$, or equivalently the scale factor $S=\left(\frac{R_{w d}}{R_{\odot}}\right)^{2}\left(\frac{d}{k p c}\right)^{-2}$, is the factor by which the theoretical flux (integrated over the entire wavelength range) has to be multiplied to equal the observed (integrated) flux.

The grid of WD models extended over the following range of parameters: $\log g=$ 7.0, 7.5, 8.0, 8.5, 9.0; $\mathrm{T}_{\text {eff }} / 1000(\mathrm{~K})=22,23, \ldots, 75 ; \mathrm{Si}=0.1,0.2,0.5,1.0,2.0,5.0 ; \mathrm{C}=0.1$, $0.2,0.5,1.0,2.0,5.0$; and $v_{\text {rot }} \sin i\left(\mathrm{~km} \mathrm{~s}^{-1}\right)=100,200,400,600,800$.

For the synthetic accretion disk models, we used the latest accretion disk models from the optically thick disk model grid of Wade \& Hubeny (1998). The range of disk model parameters varies as follows: WD mass (in solar units) value of $0.35,0.55,0.80,1.03$, and 1.21; orbital inclination (in degrees) of $18,41,60,75$ and 81 . The accretion rate ranges from $10^{-10.5} M_{\odot \mathrm{yr}^{-1}}$ to $10^{-8.0} M_{\odot} \mathrm{yr}^{-1}$ by increments of 0.5 in $\log \dot{M}$.

For each dwarf nova, we adopted the following procedure. First, we masked out all of 
the obvious emission features and artifacts in both the FUSE and IUE spectra of each object. Second, we carried out synthetic spectral fits using and/or combining model components in this order: a white dwarf model alone, accretion disk model alone, combination white dwarf plus accretion disk model, and two-temperature white dwarf model (the latter to simulate a hotter equatorial region as well as a cooler photosphere at higher latitudes). For accretion disk fits, we "fine-tuned" the derived accretion rate of the best-fitting disk model by changing the accretion rate in increments of 0.1 over the range 0.1 to 10 , on the assumption that the disk fluxes scale linearly over that range.

In Table 4, we indicate where we masked any strong emission features, artifacts, or negative fluxes in the FUSE and IUE spectra of each object.

\section{Synthetic Spectral Fitting Results}

The noise level of the FUSE spectra precludes the opportunity to extract reliable parameters for the accreting white dwarfs in these three systems. This is especially true for deriving rotational velocities which rely on well-resolved, strong absorption lines arising in the photosphere. The rotational velocity is also affected by underlying emission filling of absorption features and by the chemical abundances one uses. With these caveats in mind, we proceeded to apply our grid of WD photosphere models (keeping the chemical abundance fixed at solar) and accretion disk synthetic spectra.

For any dwarf nova in quiescence, a single temperature white dwarf model should be a reasonable first approximation as the source of the FUV flux. For UU Aql, we adopted two possible distances, $150 \mathrm{pc}$ and $350 \mathrm{pc}$ and carried out detailed fits for both values. For a distance of $350 \mathrm{pc}$, the best-fit WD model to the FUSE spectrum gave $\mathrm{T}_{\text {eff }}=27,000 \mathrm{~K}$, $\mathrm{R}_{w d} / \mathrm{R}_{\odot}=1.13 \times 10^{-2}$ and a $\chi^{2}=0.963$. This best-fitting model is shown in figure 4 . The continuum of the model gives a fair representation of the observed continuum down to about $1060 \AA$ but there is a shortfall of model flux relative to the data at wavelengths shorter than $1030 \AA$. For the same distance, an accretion disk alone yielded a best fit with $\chi^{2}=1.14$, an accretion rate of $5 \times 10^{-11} M_{\odot} / \mathrm{yr}$ and inclination $i=41$ degrees, $\mathrm{M}_{w d}=0.8 M_{\odot}$. This disk fit is shown in figure 5. The model disk continuum, unlike the WD, fails to match the flux

level of the data between about $1090 \AA$ and $1180 \AA$ and the solar abundance accretion disk model fails to provide a sharp absorption features.

A combination white dwarf plus accretion disk yielded a modest improvement with $\chi^{2}=0.71, \dot{M}=1.6 \times 10^{-11} M_{\odot} / \mathrm{yr}, i=41$ degrees and $M_{w d}=0.8 M_{\odot}$. Finally we tried two temperature (WD + belt) fits. The best fit two temperature WD yields $\chi^{2}=0.74$ with the 
cooler white dwarf portion $\left(\mathrm{T}_{\text {eff }}=24,000 \mathrm{~K}\right)$ giving $57 \%$ of the flux and the hotter belt $\left(\mathrm{T}_{\text {belt }}=33,000 \mathrm{~K}\right)$ providing $43 \%$ (figure 6 ).

In general, for a distance $\mathrm{d}=150 \mathrm{pc}$, the model fits to UU Aql's FUSE spectrum are worse than for $350 \mathrm{pc}$. Applying single temperature white dwarf fits, we obtained $\mathrm{T}_{\text {eff }}=$ $17,000 \mathrm{~K}, R_{w d} / R_{\odot}=1.06 \times 10^{-2}$ and a $\chi^{2}=1.13$. An accretion disk alone yielded a best fit with $\chi^{2}=1.23$, an accretion rate of $1.3 \times 10^{-11} M_{\odot} /$ yr and inclination $i=41$ degrees and $\mathrm{M}_{w d}=0.8 M_{\odot}$. A combination white dwarf plus accretion disk yielded a modest improvement with $\chi^{2}=1.13, \dot{M}=1.6 \times 10^{-11} M_{\odot} / \mathrm{yr}, i=41$ degrees and $M_{w d}=0.8 M_{\odot}$. A two temperature white dwarf (WD + belt) gives a best-fit with a $\chi^{2}=1.02$, with a $17,000 \mathrm{~K}$ white dwarf providing $77 \%$ of the FUV flux and the belt giving $23 \%$ of the flux. In view of the much better agreement of the models with the observations of UU Aql for our assumed distance of $350 \mathrm{pc}$ than for a distance of 150 pc, the closer distance can be ruled out.

For CH UMa, the FUSE spectrum is very noisy and underexposed. We took into account our adopted distance of $300 \mathrm{pc}$ in the model fitting, we fixed the WD mass at $\mathrm{M}_{w d}=1.2 M_{\odot}$ $(\log g=9.0$, with a radius of $\approx 4,000 \mathrm{~km})$, and fixed the disk inclination at the published value of 18 degrees. A single temperature white dwarf fit to $\mathrm{CH} \mathrm{UMa}$ had $\chi^{2}=0.227$, and yielded a best fit $\mathrm{T}_{\text {eff }}=29,000 \mathrm{~K}$, and a distance of $310 \mathrm{pc}$. This model however did not fit very well in the shorter wavelengths $(<1020 \AA)$. A lowest $\chi^{2}$ fit for this same WD mass was obtained for $\mathrm{T}=40,000 \mathrm{~K}$, with $\chi^{2}=0.208$, but it yielded a distance of $600 \mathrm{pc}$, twice the adopted estimate of the distance. Since the Ritter catalog give a mass of $1.95 M_{\odot}$ (well above the Chandraskhar mass limit for a WD), we decided to try a larger mass with a correspondingly smaller radius which lead to a smaller emitting surface area and therefore a shorter distance. We assumed $M=1.38 M_{\odot}(\log g=9.5$, with a radius of $\approx 2,000 \mathrm{~km})$ and found that the best fit was for $T=40,000 \mathrm{~K}$. This model yielded a distance of 307pc with $\chi^{2}=0.199$. Since the absorption features around $1120 \AA-1150 \AA$ are not pronounced in the observed spectrum, we decided to increase the rotational velocity to improve the fit of this model. However, a better result was obtained by simply reducing the abundances of Si and C to 0.01 their solar value. This low Si and $\mathrm{C}$ model had a $\chi^{2}=0.184$ and a distance of $314 \mathrm{pc}$. This model is shown in Figure 7. Because of the low $\mathrm{S} / \mathrm{N}$ of the spectrum the assessment of the error on the temperature estimate is of the order of $5,000 \mathrm{~K}$, namely $T_{w d}=40,000 \pm 5,000 \mathrm{~K}$.

Though the WD fit yielded the lowest value of $\chi^{2}$, we tried accretion disk fits alone to $\mathrm{CH}$ UMa's FUSE data. The best fit gave a $\chi^{2}=0.213$, an accretion rate of $5 \times 10^{-12} M_{\odot} / \mathrm{yr}$ for $M_{w d}=1.2 M_{\odot}$ and $i=18$ degrees.

Various attempts to fit the combined FUSE + IUE spectra of CH UMa met with limited success as summarized in Table 5. The best combination fit was for an accretion disk plus 
WD with the disk contributing $79 \%$ of the FUV flux. However, these fits were less satisfactory than the fits to the FUSE spectrum alone.

Because the FUSE spectrum of CH UMa is very noisy, and because of the broad emission lines and the absence of strong absorption lines, it is difficult to assess which model (disk or WD) is the best solution. However, the absence of strong absorption lines would favor the accretion disk model because of Keplerian broadening. Also because of the poor quality of the spectrum, composite models (WD+accretion disk, two-temperature WD) did not improve the fit. In view of all of the above, we cannot be confident that we have determined the temperature of the white dwarf in $\mathrm{CH} \mathrm{UMa}$.

Next we analyzed the combined FUSE + IUE spectra of $\mathrm{CH}$ UMa. The best white dwarf-only fit yielded $\mathrm{T}_{e f f}=31,000 \mathrm{~K}, \log g=9$ a $\chi^{2}=3.02$ and $R_{w d} / R_{\odot}=6.02 \times 10^{-3}$. For accretion disk models only, the best fit occurred with a $\chi^{2}=2.02$, corresponding to $\dot{M}=3 \times 10^{-9} M_{\odot} /$ yr. This disk fit is a modest improvement of the fit to CH UMa since the $\chi^{2}$ value was lowered to 2.02 . The best-fitting white dwarf + accretion disk model resulted in only a modest, statistically insignificant improvement. The $\chi^{2}$ value was lowered to 1.87 , the accretion rate was $6.4 \times 10^{-11} M_{\odot} / \mathrm{yr}$ with the WD $\mathrm{T}_{\text {eff }}=22,000 \mathrm{~K}$ and the scale factor yielding a white dwarf radius of $5.84 \times 10^{-3} R_{\odot}$. In this composite disk plus white dwarf fit, the WD contributes $21 \%$ of the FUV flux while the accretion disk contributes $79 \%$ of the FUV flux. We also tried a two-temperature WD solutions with the best-fitting model consisting of a $26,000 \mathrm{~K}$ WD providing $77 \%$ of the flux and a hot accretion belt/ring with $\mathrm{T}_{\text {belt }}=50,000 \mathrm{~K}$ giving $23 \%$ of the UV flux. However, this two-temperature fit was no better than the WD + accretion disk fit.

For BV Cen we dereddened the spectrum assuming $\mathrm{E}(\mathrm{B}-\mathrm{V})=0.10$, and first tried single temperature white dwarf fits with a white dwarf mass $=0.83 M_{\odot}$, and used solar chemical abundances. We found the best-fitting white dwarf model to have $\mathrm{T}_{\text {eff }}=40,000 \pm 1000 \mathrm{~K}$, $\log g=8.3, V \sin i=500 \mathrm{~km} / \mathrm{s} \pm 100 \mathrm{~km} / \mathrm{s}$. This fit yielded $\chi^{2}=0.2701$ and a distance $\mathrm{d}=435 \mathrm{pc}$ (see figure 8 ). We note here that the WD solution fits the following absorption

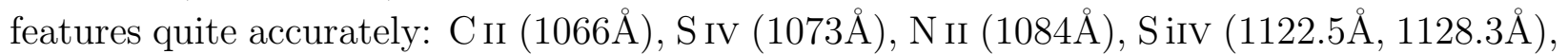

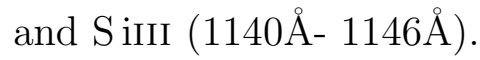

For an optically thick, steady state accretion disk alone, we chose $M_{w d}=0.80 M_{\odot}$, $i=60$ degrees. The best fit we obtained has a mass accretion rate of $10^{-8.5} M_{\odot} \mathrm{yr}^{-1}$, too large for dwarf nova quiescence. Moreover, the longer wavelength part of the spectrum is rather flat and unable to fit the absorption features around $1120 \AA$ and $1130 \AA$. This fit, with $\mathrm{i}=60 \mathrm{deg}$, leads to a distance of $1255 \mathrm{pc}$. In order to fit these absorption features, one needs to assume an inclination of 18 degrees, inconsistent with the known inclination of the system (62 degrees). Such a model leads to a distance of more than 2000pc, again inconsistent 
with all estimates of the system distance $(500 \mathrm{pc})$. Therefore, based on the parameters of the system, the disk solution is completely inconsistent. We tried composite model fits, but they also led to very poor results. We also combined BV Cen's FUSE + IUE spectra but they led to very poor fits, much worse than the fits to the FUSE spectrum alone. Since the WD solution is consistent with the parameters of the system and fits the absorption features of the spectra, it is clear that the favored solution of the FUSE spectrum of BV Cen is $T_{w d}=40,000 \pm 2,000 \mathrm{~K}$.

\section{Discussion}

Our principal objective of determining the surface temperatures of the white dwarfs in these three dwarf novae during quiescence has met with mixed results. From synthetic spectral fits to the FUSE spectra of the three long period dwarf novae, UU Aql, CH UMa and BV Cen, and the FUSE + IUE archival SWP spectra of CH UMa we have presented preliminary evidence that during quiescence, their accreting white dwarfs all have surface temperatures hotter than 20,000K. Unfortunately, all three temperatures have considerable uncertainty due to the low $\mathrm{S} / \mathrm{N}$ of the FUSE spectra and IUE spectra as well as the difficulty of disentangling the flux contribution of the second component of FUV flux or "accretion disk" during quiescence. Of the three systems in this study, we regard our estimate of the $T_{\text {eff }}$ of the WD in UU Aql to be the most reliable since that system appears to be dominated in the FUV by the white dwarf flux.

For UU Aql, we used both assumed distances, 150 pc and 350 pc, and tried the composite fits (WD + disk) to UU Aql's FUSE spectrum. However, the quality of the fits for WD-only, disk-only, WD + disk, and WD + "belt" were all roughly comparable and thus it is difficult to distinguish the best-fit case. For both distances, statistically insignificant improvements in the fits result when a white dwarf and accretion disk are combined or a two-temperature WD (WD + belt) is applied to the FUSE data. The results in Table 5 illustrate the difficulty. Qualitatively, the composite fits involving combinations of white dwarf plus accretion disk or accretion belt models look marginally more reasonable than the fits that involve single component (WD or Disk). It appears that for both distances, the white dwarf component is the dominant source of FUV flux and that the $T_{\text {eff }}$ of the WD is probably between $17,000 \mathrm{~K}$ and $27,000 \mathrm{~K}$ ( say 22,000土5,000K).

For BV Cen, the WD model fit to the FUSE spectrum gave the best result, both in fitting actual features of the observed spectrum and in leading to consistent values of the system parameters. This was not the case for the disk model and the composite models. From the best WD model fit we obtained that the WD of BV Cen must have a temperature 
of about $40,000 \mathrm{~K}$.

For CH UMa, the broad and dominant emission lines together with the poor S/N (and possible detector noise at very short wavelengths) of the FUSE and IUE spectra precluded the opportunity to obtain conclusive results. However, the results for the FUSE spectrum were of a much higher quality than for the FUSE + IUE combined spectrum. Therefore we adopt the FUSE results for CH UMa with the possibility that the WD temperature could be as high as 40,000K.

In Table 6, we list the dwarf novae above the period gap whose white dwarfs have surface temperature determinations. In Column (1) we give the system name, column (2) the orbital period; column (3) the surface temperature and column (4) the temperature reference. As seen in Table 6, there is now a sample of eight long period dwarf novae of which roughly seven have relatively secure white dwarf temperatures obtained during quiescence. In the case of BV Cen, the inclination is expected to be high. Thus, it is plausible to expect that the quiescent accretion disk may be blocking the direct radiation from the accreting white dwarf.

The relatively poor FUSE spectral quality of $\mathrm{CH}$ UMa underscores the need for reobservation with longer exposure times. However, analysis will remain hindered until more reliable information on white dwarf masses and distances becomes available. Until then, the conclusions in this work must be regarded as preliminary.

\section{Acknowledgements}

We thank an anonymous referee for helpful comments and corrections. PG wishes to thank the Space Telescope Science Institute for its kind hospitality. This work was supported by NSF grant AST05-07514 and NASA grants NAG5-12067 and NNG04GE78 to Villanova University. This research was based on observations made with the NASA-CNES-CSA Far Ultraviolet Spectroscopic Explorer. FUSE is operated for NASA by the Johns Hopkins University under NASA contract NAS5-32985. 


\section{REFERENCES}

Araujo-Betancor, S., et al. 2003, ApJ, 583, 437

Bruch, A., \& Engel, A.1994, A\&AS, 68, 41

Friend, M.T., Martin, J.S., Connon-Smith, R., \& Jones, D.H.P. 1990, MNRAS, 246, 654

Gilliland, R. 1982, ApJ, 263, 302

Godon, P., Seward, L., Sion, E.M., \& Szkody, P. 2006, AJ, 131, 2634

Harrison, T.E., Johnson, J.J., McArthur, B.E., Benedict, G.F., Szkody, P., Howell, S.B., Gelino, D.M. 2004, AJ, 127, 460

Hartley, L.E., Long, K.S., Froning, C.S., \& Drew, J.E. 2005, ApJ, 623, 425

Hubeny, I. 1988, Comput. Phys. Comm., 52, 103

Hubeny, I., \& Lanz, T. 1995, ApJ, 439, 875

laDous, C. 1991, A\&A, 252, 100

Ritter, H., \& Kolb, U. 2003, A\&A, 404, 301

Sion, E.M. 1991, AJ, 102, 295

Sion, E.M. 1999, PASP, 111, 532

Sion, E.M., Cheng, F., Godon, P., Urban, J.A., \& Szkody, P. 2004, AJ, 128, 1834

Sion, E.M., Cheng, F., Long, K.S., Szkody, P., Huang, M., Gilliland, R., Hubeny, I. 1995, ApJ, 439, 957

Sion, E.M., Cheng, F., Szkody, P., Sparks, W.M., Gänsicke, B.T., Huang, M., \& Mattei, J. 1998, ApJ, 496, 449

Sion, E.M., Szkody, P., Gänsicke, B.T., Cheng, F., LaDous, C., Hassall, B. 2001, ApJ, 555, 834

Szkody, P. 1987, ApJS, 63, 685

Townsley, D., \& Bildsten, L. 2002, ApJ, 565, 35

Townsley, D., \& Bildsten, L. 2003, ApJ, 596, L227 
Urban, J., \& Sion, E.M. 2006, ApJ, 642, 1029

Verbunt, F. 1987, A\&A, 71, 339

Wade, R.A., \& Hubeny, I. 1998, ApJ, 509, 350

Warner, B. 1995, Cataclysmic Variable Stars (Cambridge University Press) 
Table 1: Dwarf Nova Parameters

\begin{tabular}{llllllcccc}
$\begin{array}{l}\text { System } \\
\text { Name }\end{array}$ & $\begin{array}{l}P_{\text {orb }} \\
\text { (days) }\end{array}$ & $\begin{array}{l}t_{\text {rec }} \\
(\text { days })\end{array}$ & $\mathrm{V}_{q}$ & $\mathrm{~V}_{o}$ & Sec. & $\begin{array}{c}\mathrm{i} \\
(\mathrm{deg})\end{array}$ & $\begin{array}{c}M_{w d} \\
\left(M_{\odot}\right)\end{array}$ & $\begin{array}{c}E_{B-V} \\
\text { Distance } \\
(\mathrm{pc})\end{array}$ \\
\hline BV Cen & 0.610108 & 150 & 12.6 & 10.5 & G5-8V & $62 \pm 5$ & $0.83 \pm 0.1$ & 0.10 & 500 \\
CH UMa & 0.343 & 204 & 15.3 & 10.7 & K4-M0V & $21 \pm 4$ & & 300 \\
UU Aql & 0.14049 & 71 & 16.0 & 11.0 & M2-4V & & & $150-350$ \\
\hline
\end{tabular}

Table 2. FUSE Observations

\begin{tabular}{cccccccc}
\hline \hline Target & \multirow{2}{*}{ Data ID } & Aperture & Time of Observation & $\mathrm{t}_{\text {exp }}$ & $\lambda_{\text {centered }}$ & $\mathrm{S} / \mathrm{N}(0.1 \AA)$ \\
\hline \multirow{2}{*}{ UU Aql } & \multirow{2}{*}{ C1100301000 } & LWRS 30x30 & $2004-05-16$ & $13 \mathrm{~h}: 48 \mathrm{~m}: 00 \mathrm{~s}$ & $16,121 \mathrm{~s}$ & $1035 \AA$ & 5.15 \\
BV Cen & D1450301000 & LWRS 30x30 & $2003-04-13$ & $20 \mathrm{~h}: 26 \mathrm{~m}: 00 \mathrm{~s}$ & $26,545 \mathrm{~s}$ & $1035 \AA$ & 5.9 \\
CH UMa & D1450201000 & LWRS 30x30 & $2003-04-02$ & $22 \mathrm{~h}: 00 \mathrm{~m}: 00 \mathrm{~s}$ & $17,31 \mathrm{~s}$ & $1035 \AA$ & 3.6 \\
\hline
\end{tabular}

Table 3. IUE Observations

\begin{tabular}{ccccccc}
\hline \hline Target & SWP No. & Aperture & Dispersion & Date & Time 0f Mid-Exposure & texp $_{\text {ex }}(\mathrm{s})$ \\
\hline BV Cen & 26623 & Lg & Low & $08 / 16 / 85$ & $17: 17: 00$ & 14,400 \\
CH UMa & 56270 & Lg & Low & $12 / 06 / 95$ & $12: 15: 49$ & 13,800 \\
\hline
\end{tabular}


Table 4. Masked Spectral Regions

\begin{tabular}{|c|c|}
\hline Target & Emission Features \\
\hline UU Aql & $\begin{array}{l}\text { for } \lambda<1050 \AA \text { if } F_{\lambda}>6 \times 10^{-15} \mathrm{ergs} \mathrm{s}^{-1} \mathrm{~cm}^{-2} \AA^{-1} \\
\text { for } 1050 \AA<\lambda<1185 \AA \text { if } F_{\lambda}>1.8 \times 10^{-14} \mathrm{ergs} \mathrm{s}^{-1} \mathrm{~cm}^{-2} \AA^{-1} \\
975-985 \AA\end{array}$ \\
\hline CH UMa & $\begin{array}{l}\text { for } \lambda<957 \AA \text { if } F_{\lambda}>1 \times 10^{-14} \mathrm{ergs} \mathrm{s}^{-1} \mathrm{~cm}^{-2} \AA^{-1} \\
970-980,987-994,1023-1042,1071-1090,1107-1136,1167-1180,1200-1260 \text {, } \\
1285-1315,1380-420,1532-1560,1630-1650,1846-1940 \AA\end{array}$ \\
\hline BV Cen & $\begin{array}{l}\text { for } \lambda<1185 \AA \text { if } F_{\lambda}>2.5 \times 10^{-14} \mathrm{ergs} \mathrm{s}^{-1} \mathrm{~cm}^{-2} \AA^{-1} \\
1190-1220,1320-1340,1375-1415,1530-1570,1625-1650,1835-1880 \AA\end{array}$ \\
\hline
\end{tabular}

Table 5. UU Aql, BV Cen, and CH UMa Fitting Results

\begin{tabular}{|c|c|c|c|c|c|c|c|}
\hline System & Spectrum & $\begin{array}{c}\mathrm{d} \\
(\mathrm{pc})\end{array}$ & $\chi^{2}$ & $\begin{array}{c}T_{w d} \\
(103 \mathrm{~K})\end{array}$ & $\begin{array}{c}T_{\text {belt }} \\
(103 \mathrm{~K})\end{array}$ & $\begin{array}{c}\dot{M} \\
\left(M_{\odot} / \mathrm{yr}\right)\end{array}$ & $\begin{array}{c}\% \text { Flux } \\
\text { (Contribution) }\end{array}$ \\
\hline \multirow[t]{8}{*}{ UU Aql } & FUSE & 350 & 0.96 & 27 & - & - & 100(WD) \\
\hline & FUSE & 150 & 1.13 & 17 & - & - & 100(WD) \\
\hline & FUSE & 350 & 1.13 & - & - & $5 \times 10^{-11}$ & 100(Disk) \\
\hline & $F U S E$ & 150 & 1.24 & - & - & $1 \times 10^{-11}$ & 100(Disk) \\
\hline & FUSE & 350 & 0.714 & 24 & - & $2 \times 10^{-11}$ & $73(\mathrm{WD}) / 27($ Disk $)$ \\
\hline & FUSE & 150 & 1.13 & 17 & - & $3 \times 10^{-11}$ & $60(\mathrm{WD}) / 40($ Disk $)$ \\
\hline & FUSE & 350 & 0.738 & 24 & 33 & - & $57(\mathrm{WD}) / 43$ (Belt) \\
\hline & FUSE & 150 & 1.021 & 16 & 29 & - & $77(\mathrm{WD}) / 23$ (Belt) \\
\hline \multirow[t]{6}{*}{ CH UMa } & FUSE & 314 & 0.184 & 40 & - & - & $100(\mathrm{WD})$ \\
\hline & FUSE & 313 & 0.213 & - & - & $5 \times 10^{-12}$ & 100(Disk) \\
\hline & $F U S E+I U E$ & 300 & 3.02 & 31 & - & - & $100(\mathrm{WD})$ \\
\hline & $F U S E+I U E$ & 300 & 2.02 & - & - & $3 \times 10^{-9}$ & 100(Disk) \\
\hline & $F U S E+I U E$ & 300 & 1.87 & 22 & - & $6.4 \times 10^{-11}$ & $21(\mathrm{WD}) / 79$ (Disk) \\
\hline & $F U S E+I U E$ & 300 & 2.00 & 26 & 50 & - & $77(\mathrm{WD}) / 23$ (Belt) \\
\hline \multirow[t]{2}{*}{ BV Cen } & FUSE & 435 & 0.27 & 40 & - & - & $100(\mathrm{WD})$ \\
\hline & FUSE & 1255 & 0.27 & - & - & $3 \times 10^{-9}$ & 100(Disk) \\
\hline
\end{tabular}


Table 6: Surface Temperatures of White Dwarfs in Dwarf Novae above the Period Gap

\begin{tabular}{lccl}
\hline \hline System Name & $\begin{array}{c}\text { Period } \\
(\mathrm{min})\end{array}$ & $\begin{array}{c}T_{\text {eff }} \\
(\text { Kelvin})\end{array}$ & References \\
\hline BV Cen & 878.6 & $40,000:$ & This paper \\
RU Peg & 539.4 & 49,000 & Sion et al $(2004)$ \\
Z Cam & 417.4 & 57,000 & Hartlev et al. (2005) \\
RX And & 302.2 & 34,000 & Sion et al. $(2001)$ \\
SS Aur & 263.2 & 31,000 & $\underline{\text { Sion et al }}(2004)$ \\
U Gem & 254.7 & 31,000 & Sion et al. (1998) \\
WW Ceti & 253.1 & 26,000 & Godon et al. (2006) \\
UU Aql & 202.3 & $27,000:$ & This paper \\
\hline
\end{tabular}


Figure Captions

Fig. 1.- The flux $F_{\lambda}\left(\mathrm{ergs} / \mathrm{cm}^{2} / \mathrm{s} / \AA\right)$ versus wavelength $(\AA) F U S E$ spectrum of the U Gemtype dwarf nova UU Aql during quiescence. The identifications of the strongest neutral and ionized line features as well as rotational-vibrational transitions of molecular hydrogen are marked with vertical tick marks. The molecular hydrogen absorption are modestly affecting the continuum, slicing it at almost regular wavelength intervals. We identify the most prominent molecular hydrogen absorption lines by their band (Werner or Lyman), upper vibrational level (1-16), and rotational transition (R,P, or Q) with lower rotational state $(\mathrm{J}=1,2,3)$. Air glow line features are indicated with the earth symbol. The C III $(977 \AA)$ and O VI doublet sharp emissions are due to heliocoronal emission often present in some of the FUSE channels when the target (i.e. UU Aql) is faint.

Fig. 2.- The flux $F_{\lambda}\left(\mathrm{ergs} / \mathrm{cm}^{2} / \mathrm{s} / \AA\right)$ versus wavelength $(\AA) F U S E$ spectrum of the U Gemtype dwarf nova BV Cen during quiescence. The identifications of the strongest neutral and ionized line features as well as rotational-vibrational transitions of molecular hydrogen are marked with vertical tick marks. Air glow line features are indicated with the earth symbol. The O I emissions are also due to air glow. The broad C III (977£) and O vi (doublet) features might be real broad emissions from the source, as these correspond to the left wing of the Lyman $\gamma \& \beta$ (respectively) and not much flux is expected there. The N I and N II sharp emission features are geocoronal.

Fig. 3.- The flux $\mathrm{F} \lambda\left(\mathrm{ergs} / \mathrm{cm}^{2} / \mathrm{s} / \AA\right)$ versus wavelength $(\AA)$ FUSE spectrum of the U Gemtype dwarf nova $\mathrm{CH}$ UMa during quiescence. The identifications of the strongest neutral and ionized line features as well as rotational-vibrational transitions of molecular hydrogen are marked with vertical tick marks. Several terrestrial line features are indicated with the earth symbol. There are some broad emission features associated with the source: we identify here the O vi doublet, C III (at $977 \AA$ and $1175 \AA$ ). There could be some N IV emission in the short wavelengths (between $920 \AA$ and $925 \AA$ ), but the increase of flux in the very short wavelengths suggests that most the flux at $\lambda<930 \AA$ could be higher orders of the hydrogen Lyman series associated with air glow contamination and possibly some detector noise.

Fig. 4.- A single temperature WD fit to the FUSE spectrum of UU Aql for a distance of 350 pc. The best-fit yielded $\mathrm{T}_{\text {eff }}=27,000 \mathrm{~K} \pm 3000 \mathrm{~K}, \log g=9$. The white dwarf contributes $100 \%$ of the FUV flux.

Fig. 5.- An accretion disk-only fit to the FUSE spectrum of UU Aql for a distance of 350 pc. The best-fit corresponded to $\mathrm{M}_{w d}=0.8 M_{\odot}, i=41$ degrees, $\dot{M}=5 \times 10^{-11} M_{\odot} / \mathrm{yr}$. The accretion disk contributes $100 \%$ of the FUV flux. 
Fig. 6.- A WD + belt fit to the FUSE spectrum of UU Aql for a distance of 350 pc. The dotted line is the white dwarf flux component, the dashed line is the belt flux component and the solid line is the combined two-temperature fit. This best fit two temperature WD yields $\chi^{2}=0.74$ with the cooler white dwarf portion $\left(\mathrm{T}_{\text {eff }}=24,000 \mathrm{~K}\right)$ giving $57 \%$ of the flux and the hotter belt $\left(\mathrm{T}_{\text {belt }}=33,000 \mathrm{~K}\right)$ providing $43 \%$.

Fig. 7.- A single temperature WD fit to the FUSE spectrum of $\mathrm{CH}$ UMa. The bestfit yielded $\mathrm{T}_{\text {eff }}=40,000 \pm 1,000 \mathrm{~K}, \log g=9.5$ and a projected rotational velocity of $V_{\text {rot }} \sin i=200 \mathrm{~km} / \mathrm{s}$. The portions of the spectrum that have been masked are shown in blue and include emission lines (either intrinsic to the source or due to air glow) and ISM molecular hydrogen absorption lines (which have been matched using a simple ISM model to identify the exact location of the ISM lines). Therefore the fit is between the model and the red portions of the observed FUSE spectrum. The absence of strong absorption features in the lower panel were modeled assuming low Si and C abundances and led to a lower $\chi^{2}$ than the same model with solar $\mathrm{C}$ and $\mathrm{Si}$ abundances but with a high projected rotational velocity $(\approx 1,000 \mathrm{~km} / \mathrm{s})$.

Fig. 8.- A single temperature WD fit to the FUSE spectrum of BV Cen. The best-fit yielded $\mathrm{T}_{\text {eff }}=40,000 \mathrm{~K} \pm 1000 \mathrm{~K}$, a projected rotational velocity of $500 \mathrm{~km} / \mathrm{s}$, and $\mathrm{d}=435 \mathrm{pc}$, assuming $M=0.83 M_{\odot}$.

the FUSE plus IUE spectrum of UU 


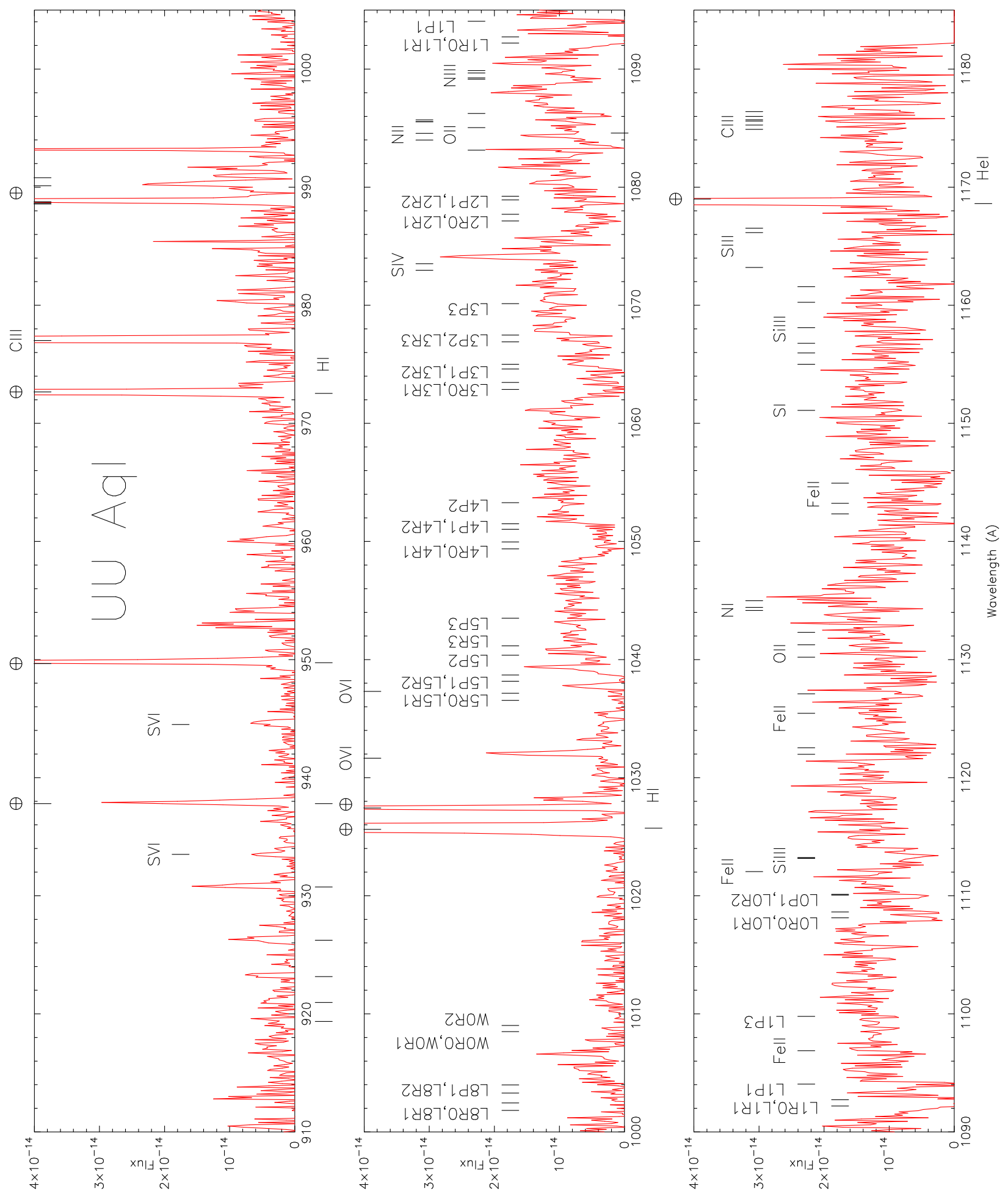




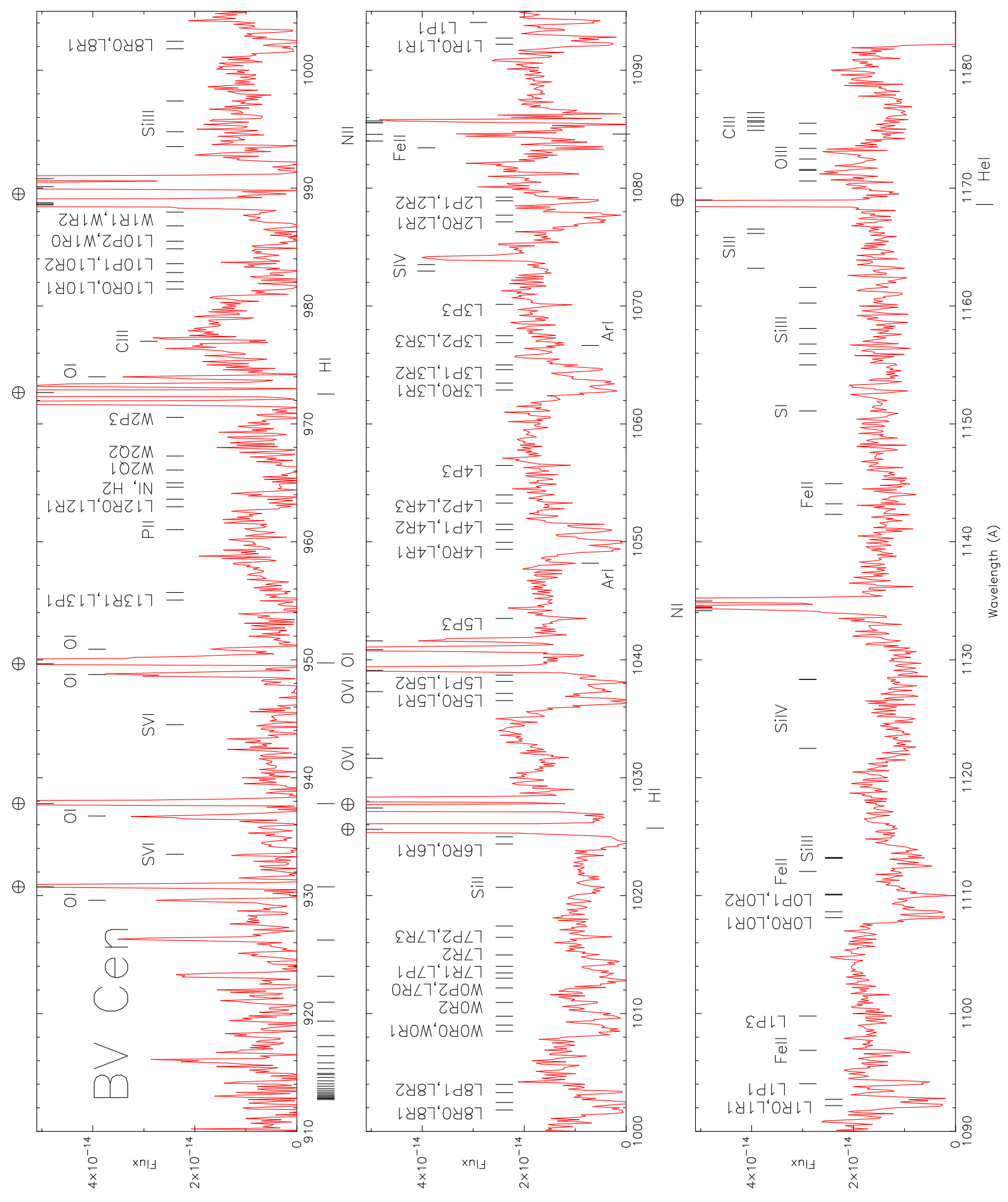




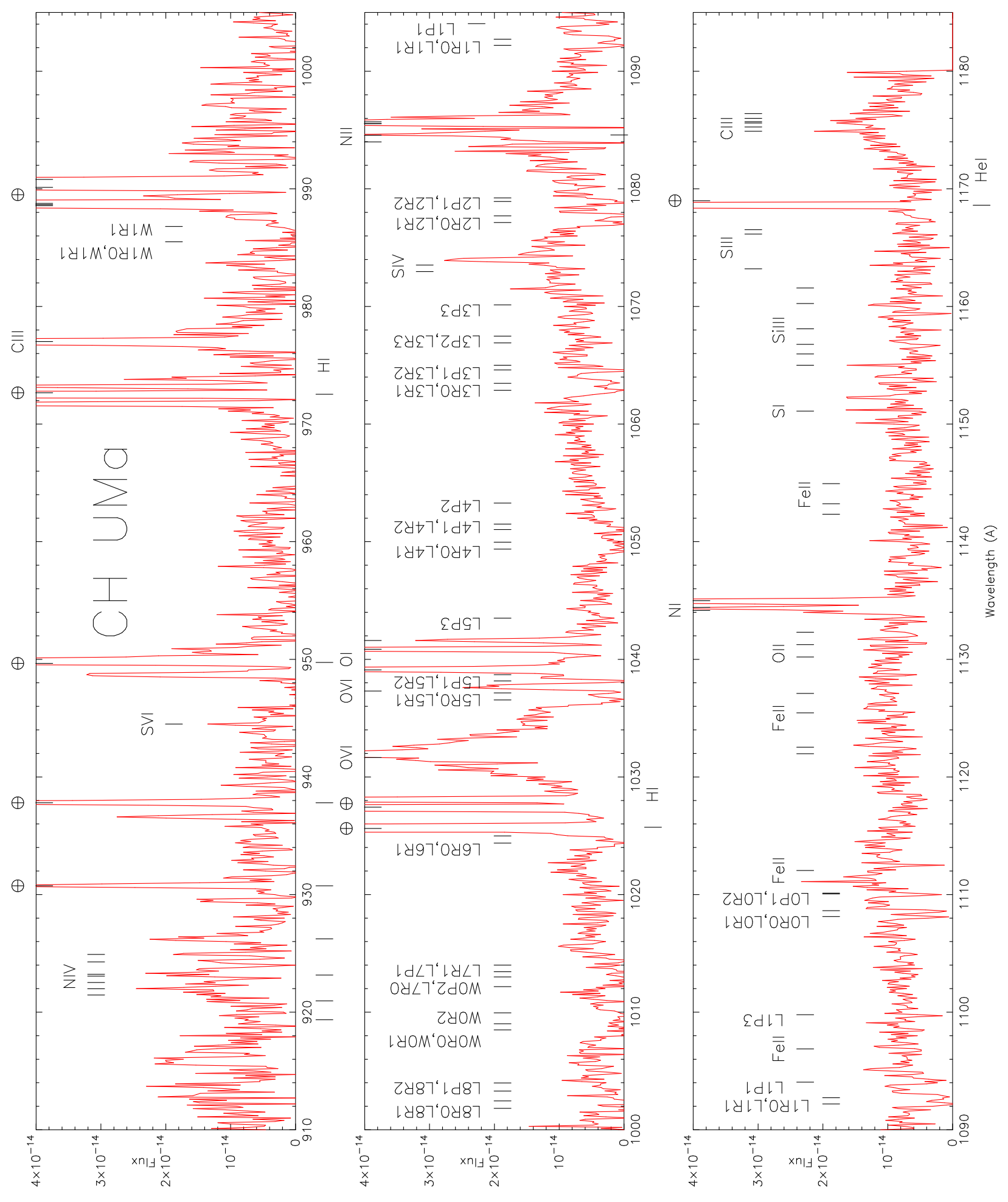




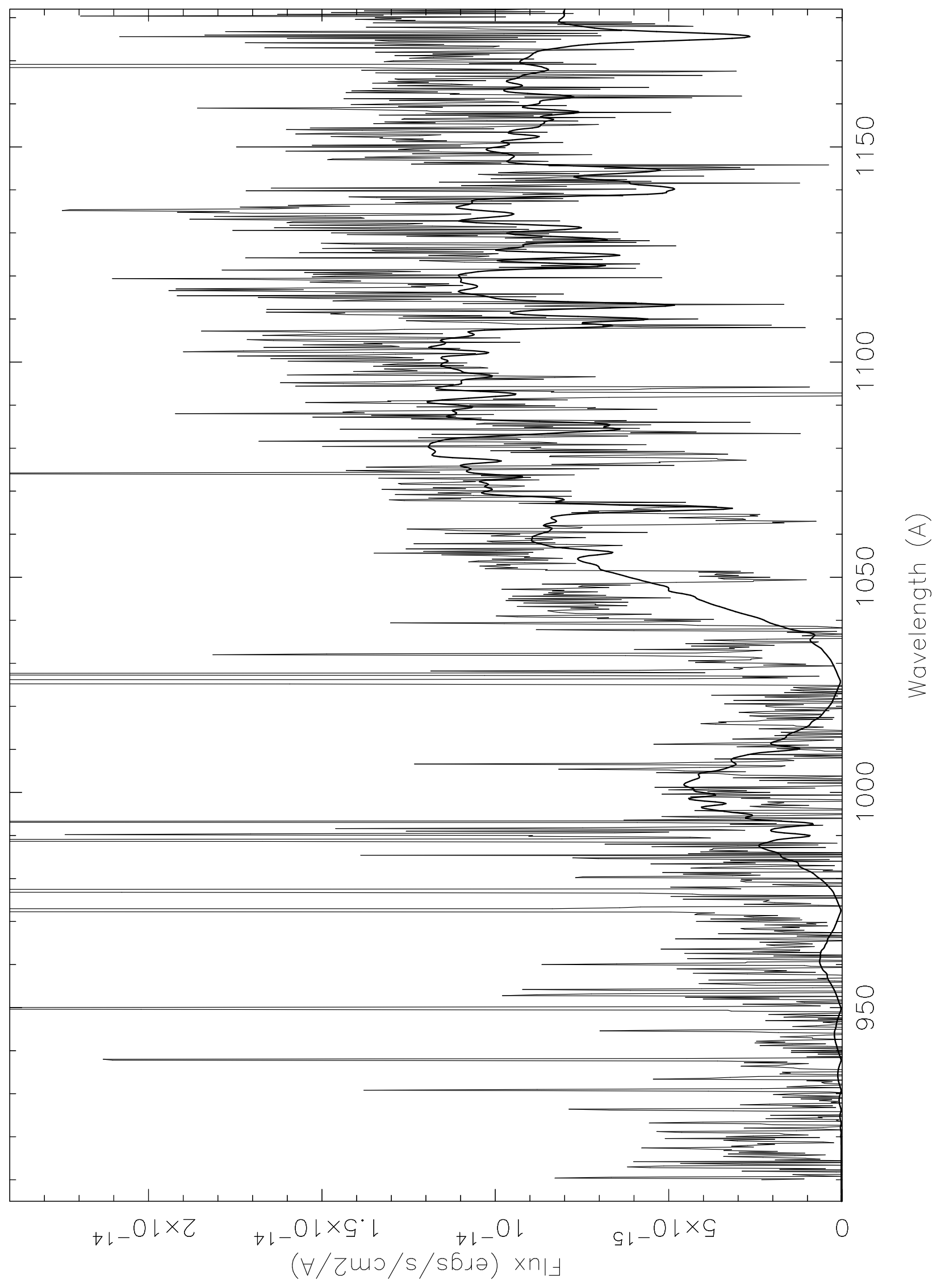




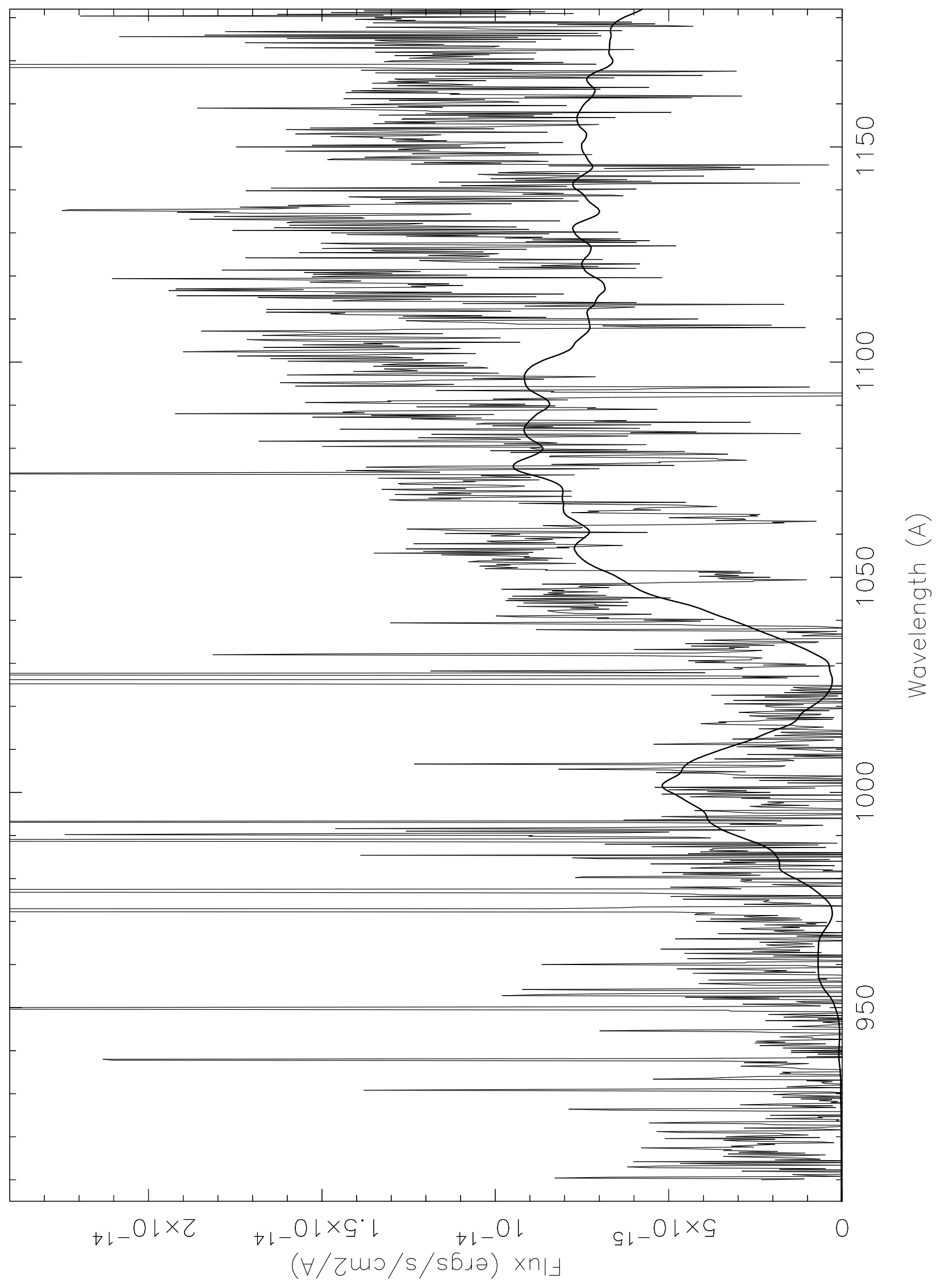




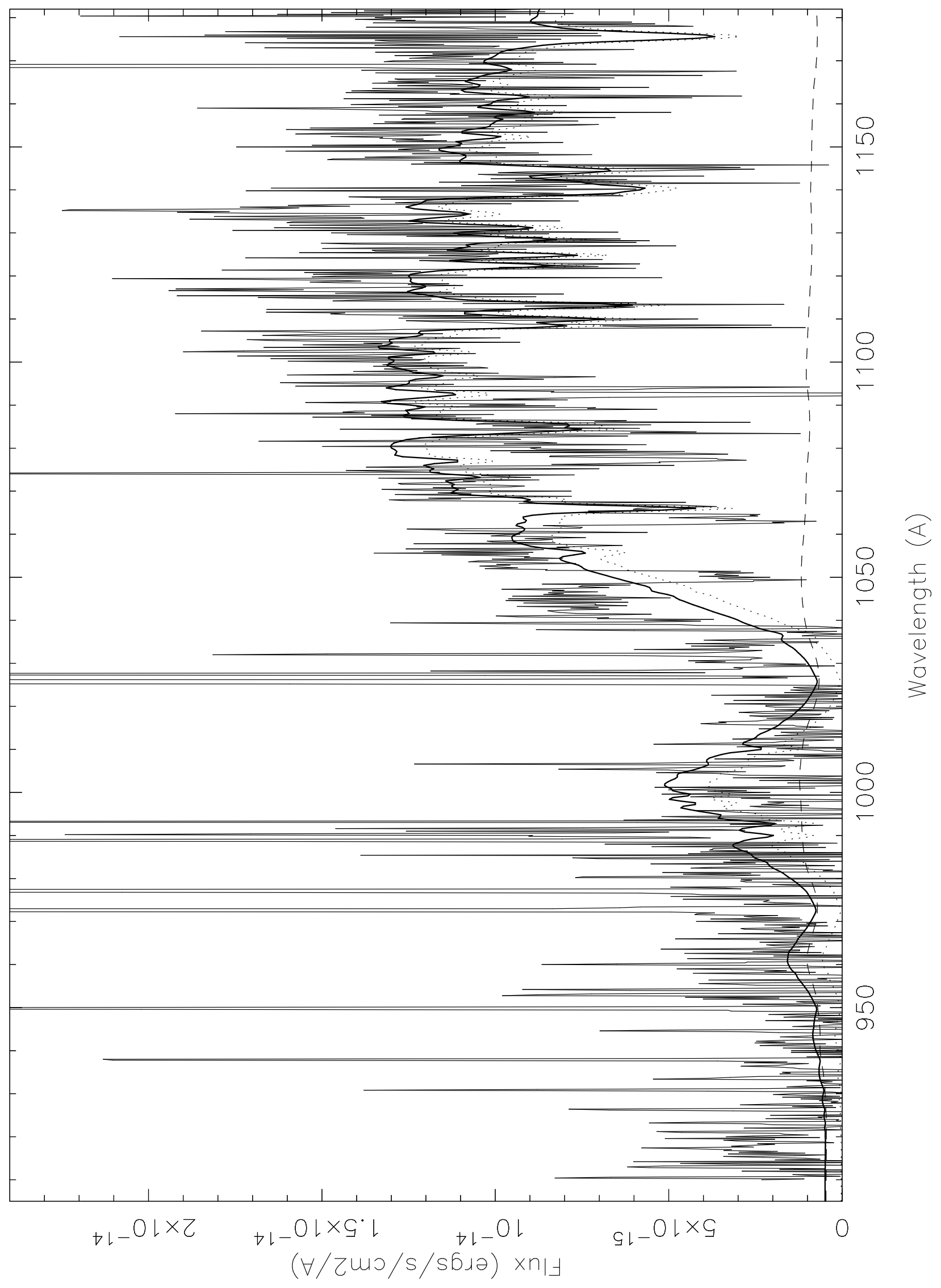




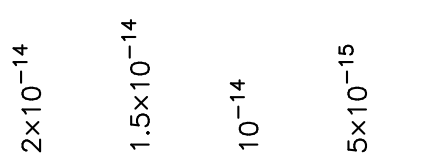

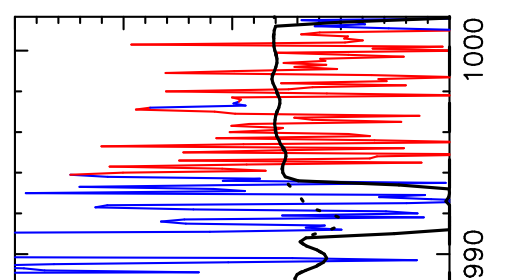

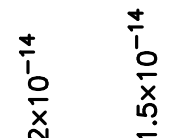

$\frac{n}{1} \frac{n}{\circ}$

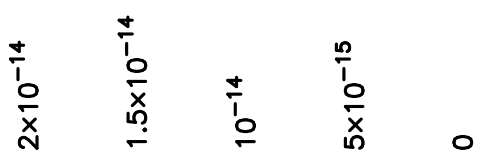
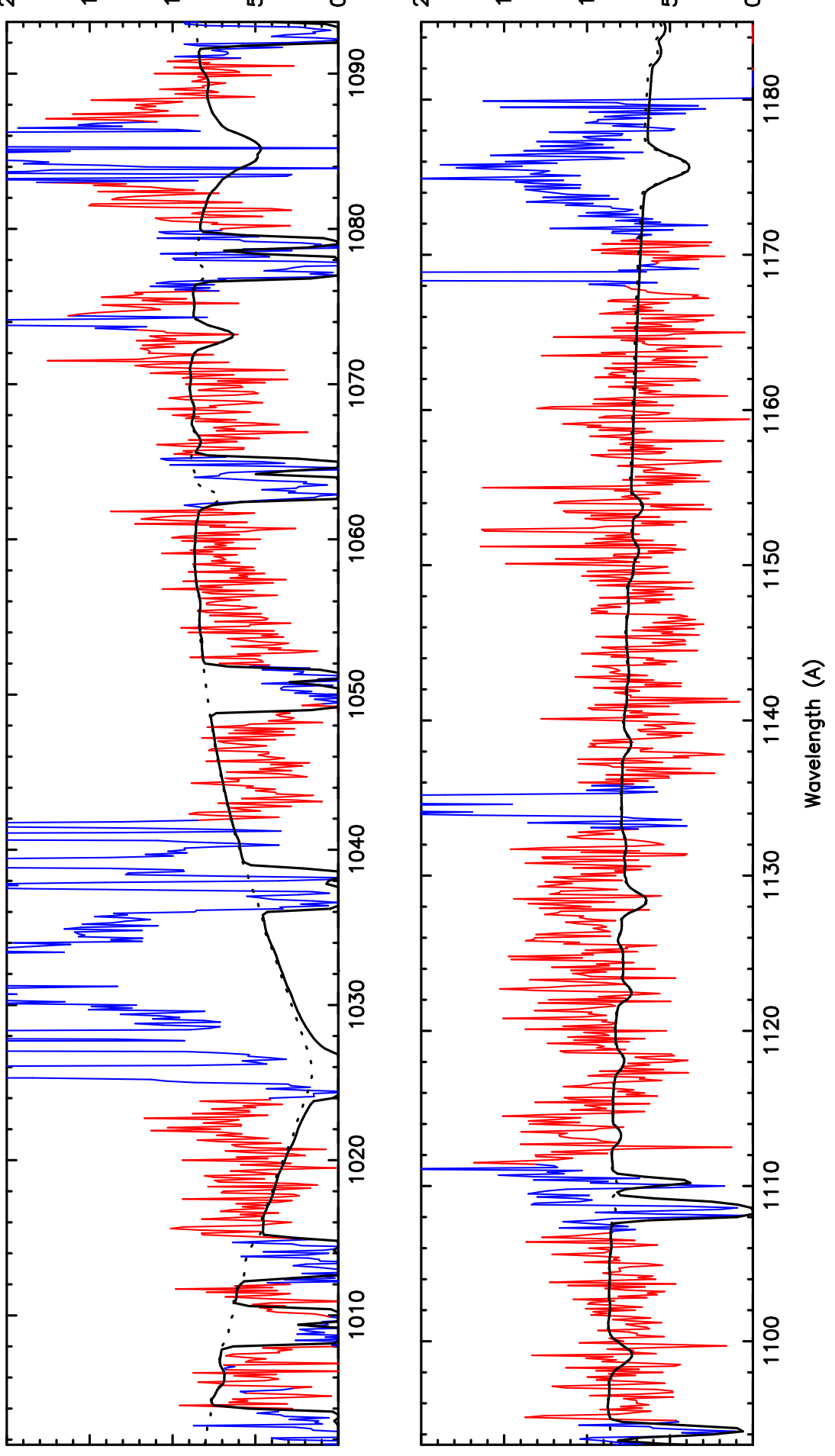

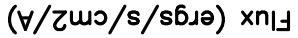




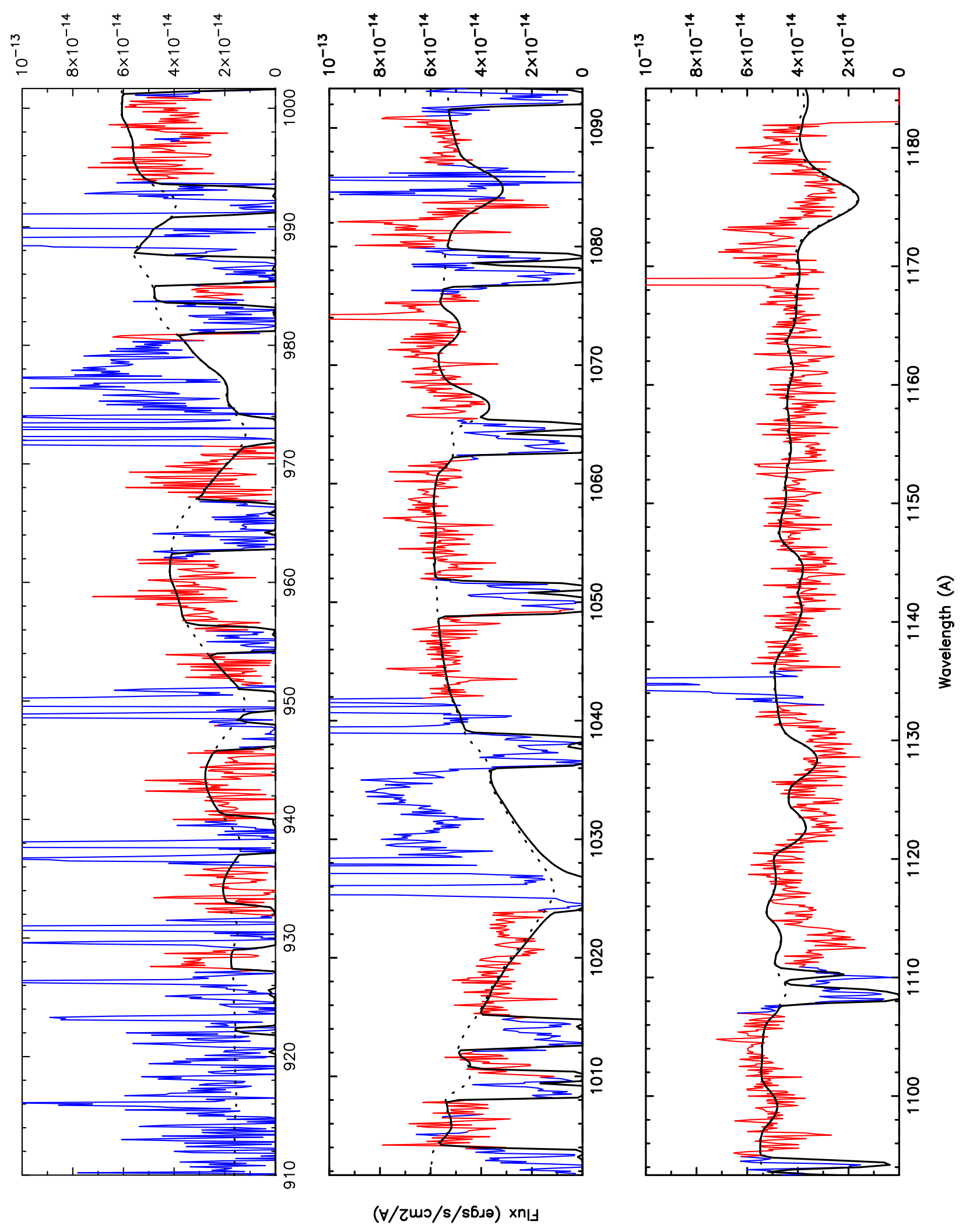

